\section{Darknet Usage in the Illegal Wildlife Trade}

\section{Issue}

The darknet is a network of websites that can be accessed only via special software that hides the details of the user's connection, and also allows websites to be hosted without revealing their location or operator. The vast majority of such 'hidden services use the Tor anonymous network (torproject.org; Dingledine et al. 2004), developed and operated by volunteers as a tool for freedom of expression and access to the internet under restrictive regimes. In addition, however, the Tor network also allows the hosting of illegal marketplaces, most famously the "Silk Road" - an eBaystyle marketplace for drugs and other illicit goods that, at its peak in 2012, was processing an estimated $\$ 15$ million in transactions annually.

Whilst the original Silk Road was shut down by law enforcement in October 2013, numerous similar markets opened in its wake. Today, large-scale darknet marketplaces exist for illegal drugs, firearms, hacking tools, stolen identity documents, and a wide variety of other illicit goods. These marketplaces typically make use of cryptocurrencies such as Bitcoin or Monero that allow decentralised electronic transactions whilst being hard to trace for law enforcement.

The untraceable nature of darknet marketplaces protect both buyers and sellers from action by law enforcement. Despite this, there have been a number of highprofile shutdowns of marketplaces, with seizure of computers and the arrest of operators, as well as numerous scams in which untraceable marketplace owners have closed marketplaces and absconded with significant amounts of untraceable funds. However the ease of use of such markets, the range of products, and the relatively low risk faced by both buyers and sellers from law enforcement have made them an attractive choice for a range of illegal goods.

The darknet has not, to date, proven to be a particularly attractive platform for the buying and selling of illegal wildlife products. Initial studies (Roberts \& Hernandez-Castro 2017) have shown that illegal wildlife products are more likely to be counterfeit goods, with the inclusion of restricted species in their manufacture a secondary factor to their value to buyers. Despite this, the darknet provides a 'marketplace of last resort' that becomes increasingly attractive over other, more accessible, online services as law enforcement and platform operators enforce policies against trading in illegal wildlife products. This makes the ongoing study of darknet markets an important avenue for research as other policies against online illegal wildlife trading emerge.

\section{Findings}

Recent work by Roberts and Castro (2017) has highlighted that there is not a large volume of specific illegal wildlife trade on the darknet, and characterise the majority of products found there as "bycatch" - products that are sold for other reasons, but that happen to contain illegal wildlife products. Key examples from the study of a year's activity on the defunct Alphabay market are the sale of hallucinogenic cacti to the drug market, and counterfeit Chanel bags produced from reptile skin.

There have, however, been a small number of more explicitly illegal products discovered on the darknet. One Alphabay seller offered both rhino horn and elephant ivory in addition to

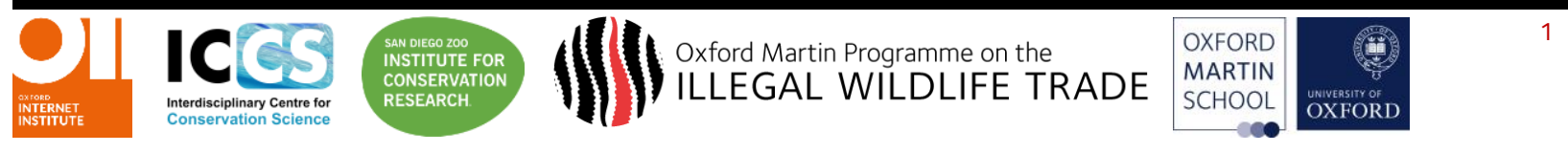


their more normal range of drugs. Similarly, a number of short-lived marketplaces have specifically been set-up to offer products from Africa, which have included gold and diamonds in addition to rhino horn.

Focusing on the usage of darknet markets, Dittus et al. (2018) studied the economic geography of seller and buyer locations as listed on five key darknet markets in 2017. These results highlighted that darknet markets typically replace the 'last mile' of the illegal drug trade - sellers are typically located geographically close to buyers in mainly consumer countries, rather than close to centres of drug production. By extensions, the flow of sales on the darknet is typically in-country, or in-continent, rather than international. This suggests that darknet markets currently replace only part of the overall supply chain for illicit goods, relying on other distribution channels for bluk transactions. Darknet markets were also shown to be largely a European and North American phenomenon, dominated by the US, the UK, and Germany, with other regions representing a significant minority of sales.

\section{Recommendations}

The darknet is characterised by substantial turnover of marketplaces, many of which last less than a year before being shut down. A key recommendation is to maintain the ongoing capacity to track emerging markets, the geographic locations that they serve, and the range of products on offer. Whilst many such marketplaces use off-the-shelf auction software, each requires manual effort to automate 'scraping' tools that monitor transactions, requiring ongoing effort.
An attractive feature of darknet markets for investigators is that the level of untraceability given by the underlying technologies makes them both relatively easy to scrape in their entirety, and largely public in the products that are offered. As such, darknet markets provide a potentially attractive means to understand shifts in consumer behaviour without the necessity of accessing closed social-media groups or forums. Monitoring the products available, and the prices at which they sell, is a second recommendation.

The darknet has significant potential for the illegal wildlife trade, despite the current low volumes shown in recent studies. With increasing adoption of technologies such as cryptocurrencies, however, and growing international enforcement efforts to prevent the trade in illegal wildlife products, the darknet is a proven venue for both high- and low-value illicit products.

\section{Key references}

Roberts D \& Hernandez-Castro J. 2017. Bycatch and illegal wildlife trade on the dark web. Oryx, 51(3), 393-394.

Dittus et al. 2018. Platform criminalism: the last-mile geography of the darknet market supply chain. Association for Computing Machinery. 277-286.

Dingledine et al. 2004. Tor: the secondgeneration onion router. SSYM'O4 Proceedings of the 13th conference on USENIX Security Symposium, 13: 21.

\section{Suggested Citation}

Wright J. 2019. Darknet Usage in the Illegal Wildlife Trade. Tools and Guidance, Oxford Martin Programme on the Illegal Wildlife Trade, University of Oxford. DOI: 10.31235/osf.io/fgrgd

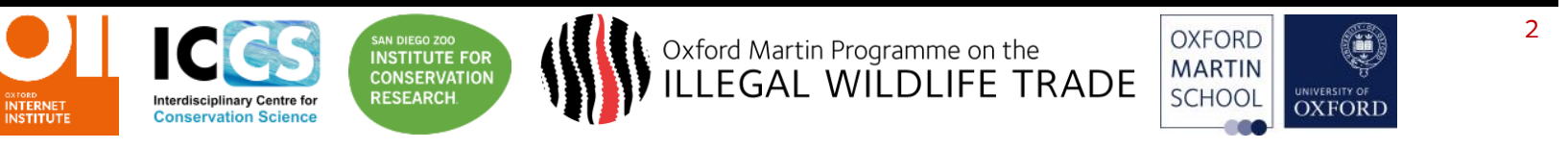

